\title{
Article
}

\section{A combination of astragaloside I, levistilide $A$ and calycosin exerts anti-liver fibrosis effects in vitro and in vivo}

\author{
Tao GUO ${ }^{1,3}$, Zu-long $\mathrm{LIU}^{1, *}$, Qiang ZHAO ${ }^{1}$, Zhi-min ZHAO ${ }^{1,2}$, Cheng-hai $\mathrm{LIU}^{1,2, *}$ \\ ${ }^{1}$ Institute of Liver Diseases, Shuguang Hospital affiliated to Shanghai University of Traditional Chinese Medicine, Shanghai 201203, \\ China; ${ }^{2}$ Shanghai Clinical Key Laboratory of Traditional Chinese Medicine, Shanghai 201203, China; ${ }^{3}$ Department of Gastroentology, \\ Hospital No.455 of the PLA, Shanghai 200052, China
}

\begin{abstract}
Liver fibrosis is excessive accumulation of extracellular matrix proteins that results from various chronic liver diseases. Hepatic stellate cells (HSCs) play an essential role in the pathogenesis of liver fibrosis. Danggui Buxue Tang (DBT) is a classic formula of Chinese traditional medicine. We previously showed that DBT could ameliorate liver fibrosis in rats. However, the bioactive components of DBT in the treatment of liver fibrosis remain unknown. In this study we evaluated 14 ingredients from DBT in human hepatic stellate cell line LX-2, and found that astragaloside I (A), levistilide $A(L)$ and calycosin $(C)$ produced synergistic proliferation inhibition on LX-2 cells and TGF- $\beta 1$-activated LX-2 cells. Thus, we prepared a mixture of them, and named this combination as ALC formula. Using high-content screening and Western blot assay we revealed that the ALC formula significantly reduced the expression of $\alpha$-SMA and collagen I in LX-2 cells. The in vivo anti-fibrosis effects of ALC formula were evaluated in a liver fibrosis model in C57BL/ 6 mice established through injection of dimethylnitrosamine (DMN $2 \mathrm{mg} / \mathrm{kg}$, ip) for 4 weeks. In the third week, the mice were injected with ALC formula (astragaloside I $44.21 \mathrm{mg} / \mathrm{kg}$ per day, levistilide A $6 \mathrm{mg} / \mathrm{kg}$ per day and calycosin $3.45 \mathrm{mg} / \mathrm{kg}$ per day; ip) or sorafenib, a positive control drug ( $6 \mathrm{mg} / \mathrm{kg}$ per day, ip) for 2 weeks. We found that administration of the ALC formula markedly decreased collagen deposition, hydroxyproline (Hyp) content and $\alpha$-SMA expression levels in the liver tissues compared to the model mice. In conclusion, the present study demonstrates for the first time that astragaloside I, levistilide A and calycosin may be the 3 main bioactive components in DBT; their combination exerts anti-liver fibrosis effects in vitro and in vivo.
\end{abstract}

Keywords: Danggui Buxue Tang; traditional Chinese medicine; astragaloside I; levistilide A; calycosin; hepatic stellate cells (HSCs); liver fibrosis

Acta Pharmacologica Sinica (2018) 39: 1483-1492; doi: 10.1038/aps.2017.175; published online 31 May 2018

\section{Introduction}

Liver fibrosis is the scarring process that results from kinds of chronic liver diseases, such as viral hepatitis, alcoholic liver disease, non-alcoholic steatohepatitis, and autoimmune diseases etc, with the characteristics of increased production of extracellular matrix (ECM) components, particularly collagens in liver ${ }^{[1-2]}$. Hepatic stellate cells (HSCs) play a central role in the pathogenesis of hepatic fibrosis as they secrete the vast majority of ECM components. Activation of HSCs is controlled by a wide variety of cytokines, growth factors, and other molecules, such as reactive oxygen species ${ }^{[3]}$. Among

\footnotetext{
*To whom correspondence should be addressed.

E-mail liuzulong@126.com (Zu-long LIU); chenghailiu@hotmail.com (Cheng-hai LIU)
}

Received 2017-07-12 Accepted 2017-11-18
HSC-activating molecules, transforming growth factor-beta 1 (TGF- $\beta 1$ ) is the most important cytokine and is responsible for HSC activation and proliferation. Activated HSCs are proliferating cells that secrete extracellular matrix proteins, including collagen type I and alpha-smooth muscle actin (a-SMA). HSCs are a crucial cell type in the injured liver, and their activation is associated with specific cytoskeletal and phenotypic profiles $^{[4]}$. Therefore, inhibition of HSC activation has been proposed as a strategy to treat liver fibrosis.

Traditional Chinese Medicine (TCM) has potential use for the treatment of chronic diseases ${ }^{[5]}$. In addition, TCM has been an accepted practice in medical care throughout East Asia for thousands of years. Our previous study found that Danggui Buxue Tang (DBT) or Danggui Buxue Decoction - an ancient traditional Chinese herbal formula composed of Huangqi (Radix Astragali) and Danggui (Radix Angelica sinensis) with 
a weight ratio of 5:1-has proven to be effective in treating liver fibrosis, and the key molecular mechanism has been revealed to be related to the prevention of degradation of lipid peroxidation as well as inhibition of MMP-2/9 activities and anti-angiogenesis ${ }^{[6-7]}$. DBT not only has a clear effect on liver fibrosis but also affects pulmonary fibrosis and renal fibro$\operatorname{sis}^{[8-12]}$.

To study the chemical composition of DBT, Yi et al first profiled 18 markers of Radix Astragali and Radix Angelica sinensis in DBT using HPLC-DAD/ELSD and LC-ESI-MS analytical methods ${ }^{[13]}$. Zhang et al compared three different ancient DBT formulas ${ }^{[14]}$. The results showed that DBT in a Radix Astragali : Radix Angelica sinensis ratio of 5:1 has the highest amount of Radix Astragali -derived astragaloside III, astragaloside IV, calycosin and formononetin, as well as Radix Angelica sinensis-derived ferulic acid. This herbal decoction has stronger osteogenic, estrogenic, and erythropoietic effects than that generated by different herb ratios ${ }^{[14]}$. To date, chemical analysis of DBT has focused on specific markers, in particular, ferulic acid and Z-ligustilide from Radix Angelica sinensis and calycosin, formononetin and astragaloside IV from Radix Astragali ${ }^{[13,15-21]}$. However, the active components of DBT that affect liver fibrosis remain unknown. Thus, we chose fourteen ingredients from DBT that are commercially available from the Shanghai Winherb Medical S\&T Development Co, Ltd ${ }^{[22]}$ as the basis of study.

In the present study, we observed the active components of DBT on LX-2 cells and calculated the combination index using the Chou-Talalay method. We then examined the effect of the ALC formula on TGF- $\beta 1$-activated LX- 2 cells and on a DMNtreated C57BL/ 6 mouse model with a hepatic damage and fibrosis phenotype. Furthermore, to confirm the effects of the ALC formula on TGF- $\beta 1$ activation, the a-SMA level in LX-2 cells and liver tissues was evaluated.

\section{Materials and methods Materials and reagents}

Dimethylnitrosamine (DMN) of the analytical reagent grade was obtained from the Tokyo KaSei Industry Co, Ltd (Tokyo, Japan). All other chemicals and solvents used were of analytical grade and were purchased from the Shanghai National Chemicals Co, Ltd (Shanghai, China). Levistilide A, astragaloside I, calycosin and other components were all purchased from the Shanghai Winherb Medical Science Co, Ltd (Shanghai, China). The purity of these standards as determined by HPLC was more than $98 \%$. Antibodies were obtained from the following companies: a-tubulin and horseradish peroxidase (HRP)-conjugated secondary antibodies (Santa Cruz Biotechnology Inc, Texas, USA); a-SMA and other antibodies are indicated in the relevant sections. Media and reagents for cell culture were acquired from Invitrogen (Life Technologies Corporation, CA, USA). All other chemicals were purchased from Sigma Chemical Co (St Louis, MO, USA)

\section{Cell culture}

The LX-2 cell line, a spontaneously immortalized human hepatic stellate cell, was a gift from Dr Scott L FRIEDMAN (Mount Sinai School of Medicine, New York, NY, USA). LX-2 cells were cultured in Dulbecco's modified Eagle's medium (DMEM) supplemented with 10\% fetal bovine serum (FBS), $100 \mathrm{U} / \mathrm{mL}$ of penicillin, and $100 \mathrm{U} / \mathrm{mL}$ of streptomycin. LX-2 cells were grown in a humidified incubator at $37^{\circ} \mathrm{C}$ in a $5 \%$ $\mathrm{CO}_{2}$ atmosphere.

\section{LX-2 cell viability and proliferation assay}

Cell viability and proliferation assays were performed using Cell Counting Kit-8 (CCK8, Beyotime Biotechnology, Haimen, China) according to the manufacturer's protocol. Briefly, LX-2 cells $\left(3.5 \times 10^{3} / 100 \mu \mathrm{L}\right.$ per well) were plated into 96 -well plates in DMEM medium with $10 \%$ FBS for $24 \mathrm{~h}$. Levistilide A, astragaloside I, calycosin, sb431542 and other components were dissolved in DMSO at a concentration of $100 \mathrm{mmol} / \mathrm{L}$ and diluted with DMEM. Ten-fold serial dilutions were made ranging from $1000 \mu \mathrm{mol} / \mathrm{L}$ to $0.1 \mu \mathrm{mol} / \mathrm{L}$. Diluted compounds were added to cells and incubated at $37^{\circ} \mathrm{C}$ with $5 \%$ $\mathrm{CO}_{2}$ for $48 \mathrm{~h}$. The medium was removed by aspiration, and 90 $\mu \mathrm{L}$ of fresh culture medium with $10 \mu \mathrm{L}$ of CCK-8 solution was added to each well. The plates were incubated for a further $2 \mathrm{~h}$ at $37^{\circ} \mathrm{C}$, and the absorbance at $450 \mathrm{~nm}$ was measured using a microplate reader (model 550, Bio-Rad Laboratories, Hercules, CA, USA). The percentage of growth is shown relative to untreated controls. Each experiment was performed independently at least three times.

\section{LX-2 cell activation and inhibition assay}

LX-2 cells $\left(3.5 \times 10^{3} / 100 \mu \mathrm{L}\right.$ per well) were seeded into 96 -well plates in DMEM containing 10\% FBS. After $24 \mathrm{~h}$, cells were washed twice with PBS and fresh DMEM without FBS was added to the culture dish for $12 \mathrm{~h}$ of starvation. Then, various concentrations of levistilide A, astragaloside I, calycosin and other components were diluted in DMEM with $10 \mathrm{ng} / \mathrm{mL}$ TGF- $\beta 1$ and added to each well. Sb431542 was added as positive control. Cells were cultured for a further $48 \mathrm{~h}$. The medium was removed by aspiration, and $90 \mu \mathrm{L}$ of fresh culture medium with $10 \mu \mathrm{L}$ of CCK- 8 solution were added to each well. The plates were incubated for a further $2 \mathrm{~h}$ at $37^{\circ} \mathrm{C}$ and the absorbance at $450 \mathrm{~nm}$ was measured; the percentage of growth is shown relative to untreated controls. Each experiment was performed independently at least three times.

\section{Chou-Talalay method}

The Combination Index (CI) was calculated to determine whether the drugs interacted synergistically, additively, or antagonistically. CI was calculated by the following equation: $\mathrm{CI}=\mathrm{D}_{1} /\left(\mathrm{D}_{\mathrm{m}}\right)_{1}+\mathrm{D}_{2} /\left(\mathrm{D}_{\mathrm{m}}\right)_{2}+\mathrm{D}_{1} \times \mathrm{D}_{2} /\left((\mathrm{Dm})_{1} \times(\mathrm{Dm})_{2}\right)$, where $\mathrm{D}_{1}$ is the concentration of a drug necessary to achieve a particular effect in the combination; $(\mathrm{Dm})_{1}$ is the concentration of the same drug that produces an identical effect by itself; $\mathrm{D}_{2}$ is the concentration of the second drug that will produce a particular effect in the combination; and $(\mathrm{Dm})_{2}$ is the concentration of the second drug that produces an identical effect by itself. $\mathrm{CI}>1$ indicates antagonism, $\mathrm{CI}<1$ indicates synergy, and $\mathrm{CI}=1$ 
indicates additivity ${ }^{[23-26]}$. Two independent experiments were performed to obtain the $\mathrm{CI}$.

\section{Immunofluorescence for $\alpha$-SMA and collagen I}

For immunofluorescence, LX-2 cells $\left(3.5 \times 10^{3} / 100 \mu \mathrm{L} /\right.$ well $)$ were seeded into 96-well plates in DMEM containing 10\% FBS. After $24 \mathrm{~h}$, cells were washed twice with PBS and fresh DMEM without FBS was added to the culture dish for $12 \mathrm{~h}$ starvation. Then, $32 \mu \mathrm{mol} / \mathrm{L}$ astragaloside I, $9.6 \mu \mathrm{mol} / \mathrm{L}$ levistilide A, 28.8 $\mu \mathrm{mol} / \mathrm{L}$ calycosin and their combinations were added to each well with $10 \mathrm{ng} / \mathrm{mL}$ TGF- $\beta 1$. As a positive control, $10 \mu \mathrm{mol} / \mathrm{L}$ sb431542 was added. Cells were cultured for a further $24 \mathrm{~h}$. Then, LX-2 cells were washed with cold PBS twice and fixed with cold methanol: acetone (1:1) for $10 \mathrm{~min}$ on ice. After three rounds of extensive washing with PBS, cells were permeated with $0.05 \%$ saponin for $15 \mathrm{~min}$. Cells were blocked with $5 \%$ bovine serum albumin in PBS buffer for $30 \mathrm{~min}$ at room temperature before being incubated with an anti-a-SMA antibody (1:100, A2547, Sigma, USA) or anti-collagen I antibody (1:100, 234167, Merck, Germany). Cells were subsequently stained with a FITC-conjugated secondary antibody. After washing, cells were double-stained with DAPI (Beyotime Biotechnology, Jiangsu, China) to visualize the nuclei. Images were taken using a Cellomics Array Scan VTI HCS Reader (Thermo Fisher Scientific, USA), and data were analyzed using Cellomics Cell Health Profiling Bio Application Software.

\section{Western blot}

Western blot analyses were performed as described. Snap-frozen liver tissues were prepared in RIPA lysis buffer containing $150 \mathrm{mmol} / \mathrm{L} \mathrm{NaCl}, 1 \% \mathrm{NP}-40,0.1 \%$ SDS, $50 \mathrm{mmol} / \mathrm{L}$ Tris- $\mathrm{HCl}$ $\mathrm{pH}$ 7.4, $1 \mathrm{mmol} / \mathrm{L}$ EDTA, $1 \mathrm{mmol} / \mathrm{L}$ PMSF and $1 \times$ complete mini. Lysates were centrifuged at $10000 \times g$ at $4{ }^{\circ} \mathrm{C}$ for $15 \mathrm{~min}$ to separate cytosol-enriched supernatant from nuclei- and membrane-enriched pellets. The protein concentrations were determined using the BCA protein assay kit mentioned above. Equal amount of proteins were separated by $10 \%$ SDS gel electrophoresis (SDS-PAGE) under denaturing and non-reducing conditions and were transferred to a nitrocellulose membrane. Membranes were blocked with 5\% nonfat milk in TBST (20 $\mathrm{mmol} / \mathrm{L}$ Tris- $\mathrm{HCl}$, pH 7.5, $150 \mathrm{mmol} / \mathrm{L} \mathrm{NaCl}, 0.1 \%$ Tween 20) at room temperature for $1 \mathrm{~h}$ and incubated with a-SMA primary antibody (1:1,000; Sigma-Aldrich, USA) at $4{ }^{\circ} \mathrm{C}$ overnight. After washing with TBST, blots were incubated with a horseradish-coupled secondary antibody in wash buffer. Signals were developed and immunoreactive bands were visualized using an ECL kit (Upstate Biotechnology, Lake Placid, NY, USA) according to the manufacturer's instructions and were quantified using a Chemi-Doc image analyzer (Bio-Rad, Hercules, CA, USA).

\section{Induction of hepatic fibrosis in mice}

This experiment was conducted according to local ethical guidelines (Shanghai University of TCM, Shanghai, China). Male C57BL/6 mice weighing 18-22 g, at the specific pathogen free (SPF) level, were provided by Sino-British SIPPR/BK
Lab Animals (Shanghai, China). Mice were housed in a room under controlled temperature $\left(22-25^{\circ} \mathrm{C}\right)$, humidity $(46 \%-52 \%)$, and lighting (12 h artificial light and dark cycle) conditions, with free access to tap water and mouse chow. The standard diet pellets contained not less than $20 \%$ protein, $5 \%$ fiber, $3.5 \%$ fats, and $6.5 \%$ ash as well as a vitamin mixture. All mice were randomly divided into five experimental groups: normal control $(n=15)$, model control $(n=18)$, sorafenib treatment $(n=16)$, high-ALC formula $(n=16)$ and low-dose ALC formula $(n=16)$. Except for the normal control group, all mice were intraperitoneally injected with $0.5 \% \mathrm{DMN}$ in saline $(2 \mathrm{~mL} / \mathrm{kg}$ body weight) three times a week for 4 weeks. On the third week, mice were treated with the positive control drug sorafenib and the ALC formula daily for two weeks. Sorafenib $(6 \mathrm{mg} / \mathrm{kg})$ or its vehicle (Cremophor/ethanol/sterile saline $=1: 1: 3$ ) was administered by intraperitoneal (ip) injection. The dosing volume was $2 \mathrm{~mL} / \mathrm{kg}$ of body weight. The proportions of cremophor: ethanol: sterile saline were $12.5 \%$ cremophor, $12.5 \%$ ethanol, and $75 \%$ sterile saline. For animals receiving Sorafenib, the drug was first dissolved in a 50\% cremophor $/ 50 \%$ ethanol mixture, and saline was added to reach the final volume immediately prior to application. Animals treated with the vehicle alone as controls received the analogue fluid mixture without the drug. Mice in the normal control and model control were administered orally by gavage with vehicle once a day for four consecutive weeks at a dose of $2 \mathrm{~mL} / \mathrm{kg}$ of body weight. From the second week to the forth week, mice in the high-dose ALC formula group were intraperitoneally injected with 44.21 $\mathrm{mg} / \mathrm{kg}$ astragaloside I, $6 \mathrm{mg} / \mathrm{kg}$ levistilide A and $13.45 \mathrm{mg} / \mathrm{kg}$ calycosin once a day. In the group that received the low-dose ALC formula, mice were intraperitoneally injected with 22.11 $\mathrm{mg} / \mathrm{kg}$ astragaloside I, $3 \mathrm{mg} / \mathrm{kg}$ levistilide A and $6.72 \mathrm{mg} / \mathrm{kg}$ calycosin once a day. Following pentobarbital sodium anesthesia, all mice were sacrificed $24 \mathrm{~h}$ after the last injection. Serum and liver samples were harvested. For histology and synchrotron radiation, tissue specimens were fixed in buffered formalin and embedded in paraffin wax. Serum and liver samples were kept frozen at $-70^{\circ} \mathrm{C}$ until they were assayed. Cremophor EL was purchased from Sigma (Sigma-Aldrich).

\section{Serum levels of liver function}

Serum was obtained from C57BL/ 6 mouse blood and centrifuged at $4700 \mathrm{r} / \mathrm{min}$ for $15 \mathrm{~min}$ at $4^{\circ} \mathrm{C}$. The activities of serum alanine aminotransferase (ALT) and aspartate aminotransferase (AST) as well as the levels of serum albumin (Alb) were quantitated following the instructions provided by the manufacturer (Nanjing Jiancheng Bioengineering Institute, Nanjing, China), including the use of standards.

\section{Hepatic hydroxyproline content determination}

Hepatic hydroxyproline (Hyp) content was measured according to the modified method of Jamall et $a l^{[27]}$. The concentration of Hyp in each sample was determined from a standard curve, which was generated from a series of known quantities of Hyp from 0.2 to $1.6 \mu \mathrm{g}$ Hyp (Peptide Co, Japan). The Hyp content was expressed as $\mu \mathrm{g} / \mathrm{g}$ of liver wet weight. 


\section{Histopathological assessment of liver injury}

Liver specimens were fixed in a $10 \%$ formaldehyde solution and dehydrated in a graded alcohol series, embedded in paraffin blocks, and cut into 4-nm-thick slices. For histopathological examination, slices were stained using a standard hematoxylin and eosin $(\mathrm{H} \& \mathrm{E})$ procedure. To investigate the hepatic collagen deposition, Sirius red-polarization staining was performed, and red staining was considered to indicate collagen deposition. Images were analyzed with a light microscope (Olympus BX40, Japan).

\section{Western blotting analysis of $\alpha$-SMA expression}

Liver tissue was homogenized in RIPA buffer, and the homogenate was centrifuged at $12000 \times g$ for $30 \mathrm{~min}$. The protein concentrations in the supernatant were determined using a BCA protein assay kit (Pierce, USA). Protein of each liver tissue sample $(30 \mu \mathrm{g})$ was subjected to $10 \%$ SDS-PAGE electrophoresis under reducing and denaturing conditions and was transferred to the nitrocellulose membrane. The membranes were blocked in 5\% nonfat milk and Tris-buffered saline containing $0.1 \%$ Tween for $1 \mathrm{~h}$ at room temperature, followed by incubation with primary antibodies as follows: mouse anti-a-SMA (1:400 dilution) and GAPDH overnight at $4{ }^{\circ} \mathrm{C}$. The blots were washed with TBS-T three times, followed by incubation with the second antibody (anti-mouse, or anti-rabbit horseradish peroxidase-conjugated antibody) for $1 \mathrm{~h}$ at room temperature. After washing, the blots were developed with Supersignal West Pico chemiluminescent substrate and exposed to X-rays (Kodak, USA) according to the manufacturer's protocol. The density of the immunoreactive bands exposed on the films was quantified and corrected for the GAPDH signal with SmartView analysis software (Smartview, China).

\section{Statistical analysis}

All data are expressed as the mean \pm SD. Statistical tests were performed using SPSS software version 18.0. Differences between groups were assessed by nonparametric one-way analysis of variance (ANOVA), followed by the least significant difference (LSD) post-hoc test, and $P$ values lower than 0.05 were considered statistically significant.

\section{Results}

Astragaloside I, levistilide A and calycosin were the three main bioactive components for inhibiting proliferation and activation of $L X-2$ cells

To identify the bioactive composition of DBT, we conducted an LX-2 cell-based screen of the fourteen main ingredients involved in the DBT. LX-2 cells were treated with fourteen compounds at concentrations from 1 to $1000 \mu \mathrm{mol} / \mathrm{L}$. Sb431542 was used as positive control, which was known to inhibit the proliferation of LX-2 cells. The fourteen compounds, only calycosin and levistilide A inhibited the proliferation of LX-2 cells, with $\mathrm{IC}_{50}$ values of 149.93 and 30.82 $\mu \mathrm{mol} / \mathrm{L}$, respectively. The other twelve compounds showed no obvious inhibition of LX-2 cells (Table 1 ).

TGF- $\beta 1$ can activate LX- 2 cells through the TGF- $\beta$ pathway
Table 1. The $\mathrm{IC}_{50}$ and $\mathrm{EC}_{50}$ value of main ingredients from DBT.

\begin{tabular}{llll}
\hline Order & Compounds & $\mathrm{IC}_{50}(\mu \mathrm{mol} / \mathrm{L})$ & $\mathrm{EC}_{50}(\mu \mathrm{mol} / \mathrm{L})$ \\
\hline 1 & Crocin I & $>1000$ & $>1000$ \\
2 & Crocin II & $>1000$ & $>1000$ \\
3 & Hydroxysafflor Yellow A & $>1000$ & $>1000$ \\
4 & Gentiopicrin & $>1000$ & $>1000$ \\
5 & Adenosine & $>1000$ & $777.91 \pm 212.3$ \\
6 & Rutin & $>1000$ & $>1000$ \\
7 & Swertiamain & $>1000$ & $>1000$ \\
8 & Sweroside & $>1000$ & $>1000$ \\
9 & Astragaioside I & $>1000$ & $>1000$ \\
10 & Astragaloside I (A) & $>1000$ & $269.05 \pm 54.7$ \\
11 & Ononin & $>1000$ & $>1000$ \\
12 & Calycosin (C) & $149.93 \pm 16.9$ & $137.92 \pm 27.1$ \\
13 & Ferulaic Acid & $>1000$ & $>1000$ \\
14 & Levistilide A (L) & $30.82 \pm 12.2$ & $6.62 \pm 4.3$ \\
Ctrl & Sorafenib & $6.93 \pm 1.2$ & $7.68 \pm 1.9$ \\
Ctrl & sb431542 & $70.25 \pm 10.32$ & $13.88 \pm 4.21$ \\
\hline
\end{tabular}

1-14, main ingredients from DBT; sorafenib and sb431542, set as positive control. $\mathrm{IC}_{50}, 50 \%$ growth inhibition on normal LX-2 cells; $\mathrm{EC}_{50}, 50 \%$ growth inhibition on LX-2 cells activated by $10 \mathrm{ng} / \mathrm{mL}$ TGF- $\beta 1$.

to promote cell proliferation ${ }^{[28]}$. However, the TGF- $\beta$ receptor inhibitor sb431542 can antagonize the effect of TGF- $\beta 1$, and the $\mathrm{IC}_{50}$ value increased from 70.25 to $13.88 \mu \mathrm{mol} / \mathrm{L}$. Therefore, the LX-2 cell activation model can be utilized to detect the activity of compounds. Interestingly, when LX-2 cells were activated by $10 \mathrm{ng} / \mathrm{mL}$ TGF- $\beta 1$, we found that adenosine, astragaloside I (A), calycosin (C) and levistilide A (L) all inhibited proliferation of TGF- $\beta 1$-activated LX-2 cells, and their $\mathrm{EC}_{50}$ values were 777.91, 269.05, 137.92 and $6.62 \mu \mathrm{mol} / \mathrm{L}$, respectively (Table 1). Crocin I, crocin II, hydroxysafflor yellow A, gentiopicrin, adenosine, rutin, swertiamain, sweroside, astragaioside I, astragaloside I and ononin had no obvious effects on the stellate cell activation model. In light of the relatively poor activity of adenosine, we believe that astragaloside I, calycosin and levistilide A are the three most effective bioactive components of DBT. Therefore, we chose astragaloside I, levistilide A and calycosin (Figure 1) to represent DBT and named the combination the "ALC formula."

\section{The ALC formula had a potent synergistic effect that inhibits proliferation of LX- 2 cells and TGF- $\beta$ activated LX- 2 cells}

To test whether $\mathrm{A}$ and/or $\mathrm{L}$ and/or $\mathrm{C}$ exerted synergic or additive effects on LX-2 cells, the dose-effect curves of single or combined drug treatment were analyzed by the medianeffect method, where combination indexes (CI) of less than, equal to, and more than 1 indicate synergistic, additive, and antagonistic effects, respectively ${ }^{[23-26]}$. We found that $\mathrm{A}, \mathrm{L}$, and $\mathrm{C}$ at concentrations ranging from 1 to $500 \mu \mathrm{mol} / \mathrm{L}$ had CI values $<1$ (Figure 2), indicating synergic effects among the agents.

To further confirm the synergistic effect of ALC on the TGF$\beta 1$ activated LX-2 cell model, we treated cells with different concentrations of A, L, C alone and in combination and found 


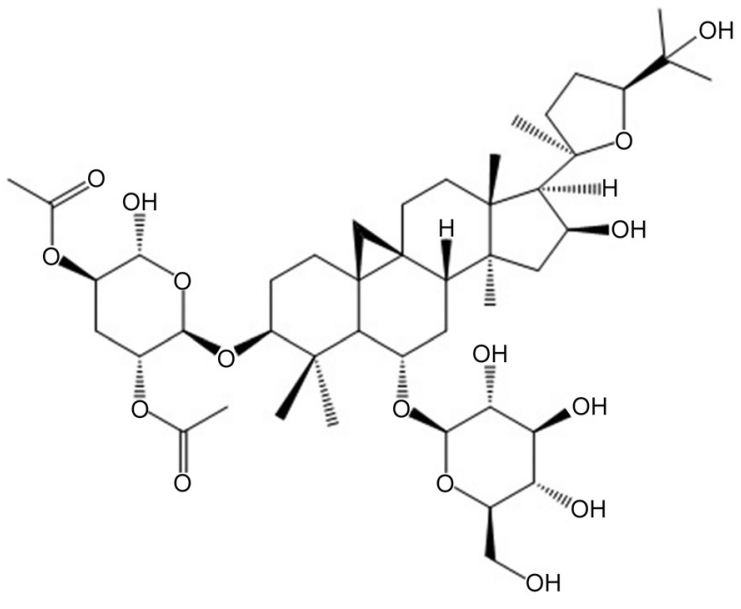<smiles>COc1ccc(-c2coc3cc(O)ccc3c2=O)cc1O</smiles>

Levistilide A (L)<smiles>CCC/C=C1\OC(=O)C2=C1CC[C@H]1[C@@H]3C=C4C(=O)O/C(=C\CCC)[C@]4(CC3)[C@H]21</smiles>

Calycosin (C)

Figure 1. The structures of Astragaloside I, Levistilide A and Calycosin.

A

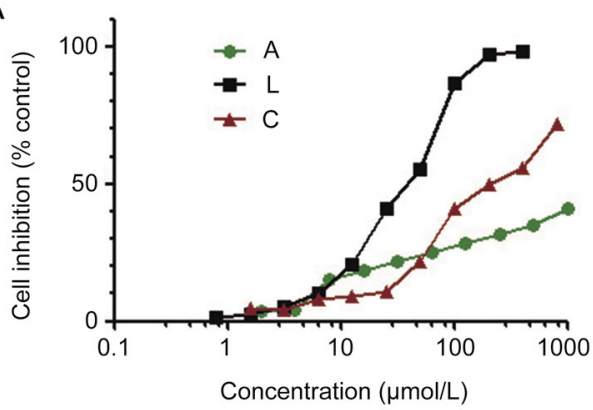

C

\begin{tabular}{cccc}
\hline Groups & a (intercept) & b (slope) & Dm $(\mu \mathrm{mol} / \mathrm{L})$ \\
\hline A & -1.37 & 0.44 & 1200.72 \\
L & -1.94 & 1.37 & 25.98 \\
C & -1.65 & 0.69 & 251.66 \\
AL & -2.04 & 1.10 & 71.16 \\
AC & -1.68 & 0.92 & 66.69 \\
LC & -1.61 & 0.81 & 94.51 \\
ALC & -2.77 & 1.46 & 78.24 \\
\hline
\end{tabular}

$\mathrm{B}$

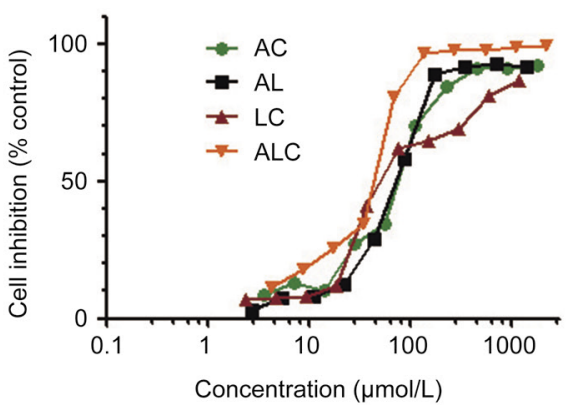

D

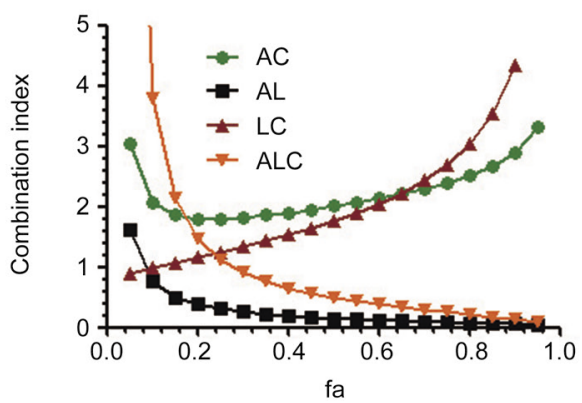

Figure 2. Synergistic effects of ALC formula on LX-2 cells. The proliferation inhibition effect of A, L, C (A) and their combination (B) exerts synergic effects on LX-2 cells, and the Dm value table (C) and combination index (D) were reflected by the median-effect method of Chou and Talalay.

that ALC also had a strong synergistic effect on the LX-2 cell activation model, as reflected by the median-effect method of Chou and Talalay (Figure 3).

\section{The ALC formula decreased expression of $\alpha$-SMA and collagen I} on LX-2

When hepatic stellate cells are activated, expression of a-SMA increases, indicating that a-SMA and collagen I are markers of HSC activation ${ }^{[29-30]}$. To further explore the effect of the ALC formula on TGF- $\beta 1$ activated LX-2 cells, we measured the expression of a-SMA and collagen I using immunofluo- rescence staining or Western blot analysis. As shown in Figure 4 , TGF- $\beta 1$ treatment significantly increased expressions of a-SMA and collagen I compared to the normal group, sb431542 inhibited TGF- $\beta 1$-induced $\alpha-S M A$ and collagen I expressions according to the fluorescence intensity of a-SMA (Figure 4A and C) and collagen I (Figure 4B and D). Among $\mathrm{A}, \mathrm{L}$, or $\mathrm{C}$ alone and in combination, we found that ALC also had a strong synergistic inhibitory effect on the fluorescence intensity of a-SMA (Figure 4A and C) and collagen I (Figure 4B and D) in LX-2 cells with effects similar to sb431542. Western blot analysis revealed that the a-SMA expression in the 
A

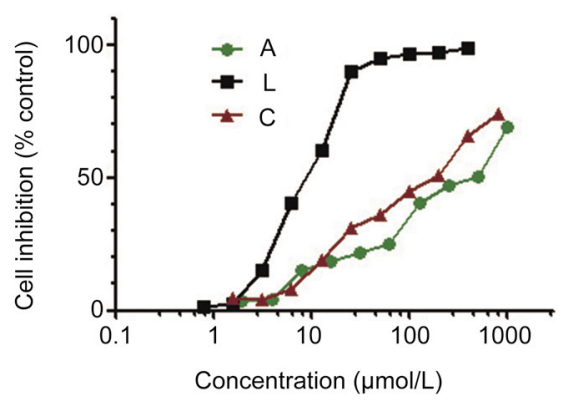

C

\begin{tabular}{cccc}
\hline Groups & $\mathrm{a}$ (intercept) & $\mathrm{b}$ (slope) & $\mathrm{Dm}(\mu \mathrm{mol} / \mathrm{L})$ \\
\hline $\mathrm{A}$ & -1.522 & 0.616 & 296.8 \\
$\mathrm{~L}$ & -1.529 & 1.469 & 11.0 \\
$\mathrm{C}$ & -1.517 & 0.701 & 146.2 \\
$\mathrm{AL}$ & -2.036 & 1.099 & 71.2 \\
$\mathrm{AC}$ & -1.675 & 0.919 & 66.7 \\
$\mathrm{LC}$ & -1.608 & 0.814 & 94.5 \\
$\mathrm{ALC}$ & -2.232 & 1.349 & 45.2 \\
\hline
\end{tabular}

B

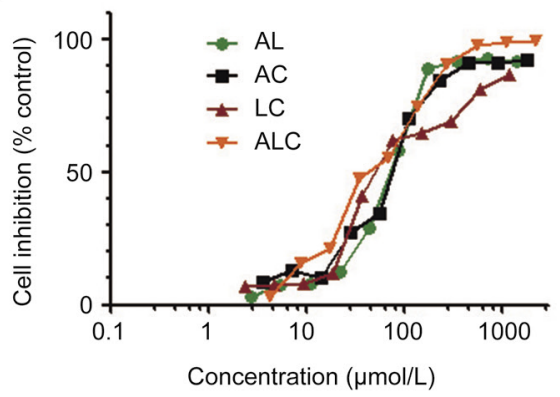

D

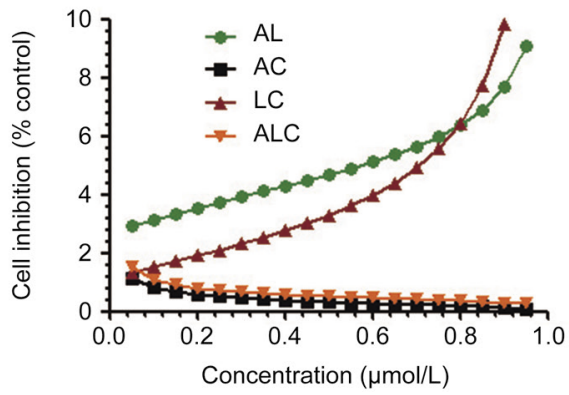

Figure 3. Synergistic effects of ALC formula on TGF- $\beta$ activated LX-2 cells. The proliferation inhibition effect of A, L, C (A) and their combination (B) exerts synergic effects on LX-2 cells, and the Dm value table (C) and combination index (D) were reflected by the median-effect method of Chou and Talalay.

model group was higher than in the normal group. However, sb431542 and the ALC formula resulted in a significant reduction in a-SMA expression (Figure $4 \mathrm{E}$ and $\mathrm{F}$ ).

In vivo assay demonstrating that the ALC formula exerted a hepatoprotective effect on dimethylnitrosamine-induced liver fibrosis

To further evaluate the in vivo anti-fibrosis effects of the ALC formula, we established a hepatic fibrosis model in C57BL/6 mice induced with an intraperitoneal injection of DMN for four weeks. From the third week, mice were treated with positive drugs and the ALC formula daily for two weeks. After mice were sacrificed at the end of the fourth week, we first detected the levels of serum liver function. As shown in Table 2, DMN intoxication resulted in significant increases in serum ALT activity $(P<0.01)$, but a lower Alb content $(P<0.05)$, in the model group compared to the normal group (AST activity was also increased, but there was no significant difference between the normal group and model group). However, the ALC formula drastically reversed the above three biomarkers compared to the model group (Table 2).

The ALC formula decreased collagen deposition and the hydroxyproline (Hyp) content in the fibrotic mouse liver

Liver fibrosis in mice was visually estimated by investigating two different histological methods, H\&E staining (Figure 5A) and Sirius red staining (Figure 5B and C). Histological analysis of livers from the control group indicated a normal liver lobular architecture with a central vein and radiating hepatic cords. The DMN group showed obvious liver inflammation,
Table 2. ALC formula improved liver function in $\mathrm{DMN}$-intoxicated mice. ${ }^{*} P<0.05,{ }^{* *} P<0.01$ vs control group. ${ }^{\#} P<0.05,{ }^{\# \#} P<0.01$ vs model group.

\begin{tabular}{llll}
\hline Groups & ALT $(\mathrm{U} / \mathrm{L})$ & AST $(\mathrm{U} / \mathrm{L})$ & Alb $(\mathrm{g} / \mathrm{L})$ \\
\hline Control & $92.98 \pm 12.90$ & $96.47 \pm 22.61$ & $26.05 \pm 3.21$ \\
Model & $200.05 \pm 42.31^{* *}$ & $124.46 \pm 41.76$ & $19.74 \pm 3.22^{\text {** }}$ \\
Sorafenib & $103.41 \pm 18.25^{\# \#}$ & $94.46 \pm 20.79$ & $20.50 \pm 2.85$ \\
ALC low dose & $95.25 \pm 11.43^{\# \#}$ & $92.20 \pm 24.16$ & $18.21 \pm 2.59$ \\
ALC high dose & $99.10 \pm 5.95^{\# \#}$ & $95.68 \pm 28.80$ & $20.11 \pm 2.38$ \\
\hline
\end{tabular}

ALC formula: astragaloside I (A), levistilide A (L) and calycosin (C); ALT: alanine aminotransferase; AST: aspartate aminotransferase; Alb: albumin.

including gross hepatocyte fatty degeneration and necrosis, broad infiltration of lymphocytes around the central vein, and marked collagen deposition, with fibrous septa and cirrhotic nodules or pseudolobules. Collagen semi-quantitative analysis showed that the collagen area of the DMN group significantly increased $(P<0.01$, Figure 5B and C). In the sorafenibtreated group, concurrent administration of sorafenib moderately improved the hepatic architecture of DMN-induced mice. Interestingly, mice treated with the ALC formula (both low- and high-dose) showed much less liver inflammation and collagen accumulation in the liver compared to the model control $(P<0.01)$ (Figure 5B and C). After mice were sacrificed, the levels of liver fibrosis in mice were also determined using the Hyp content assay. Compared to normal mice, model mice showed significant increases in Hyp content. The ALC formula (both low- and high-dose) significantly decreased the hepatic levels of Hyp $(P<0.01)$ (Figure 5D). 

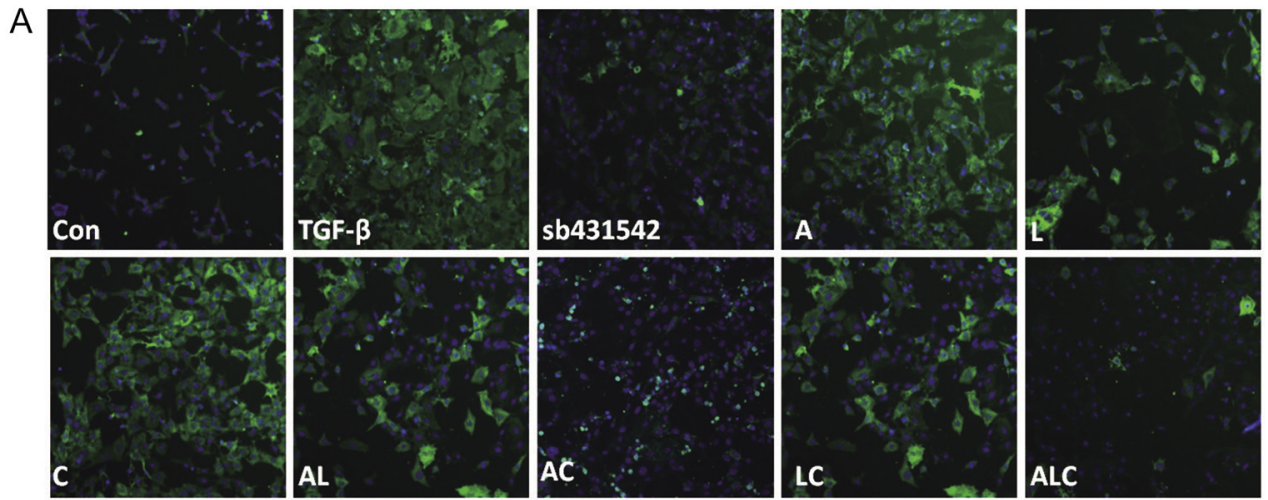

B
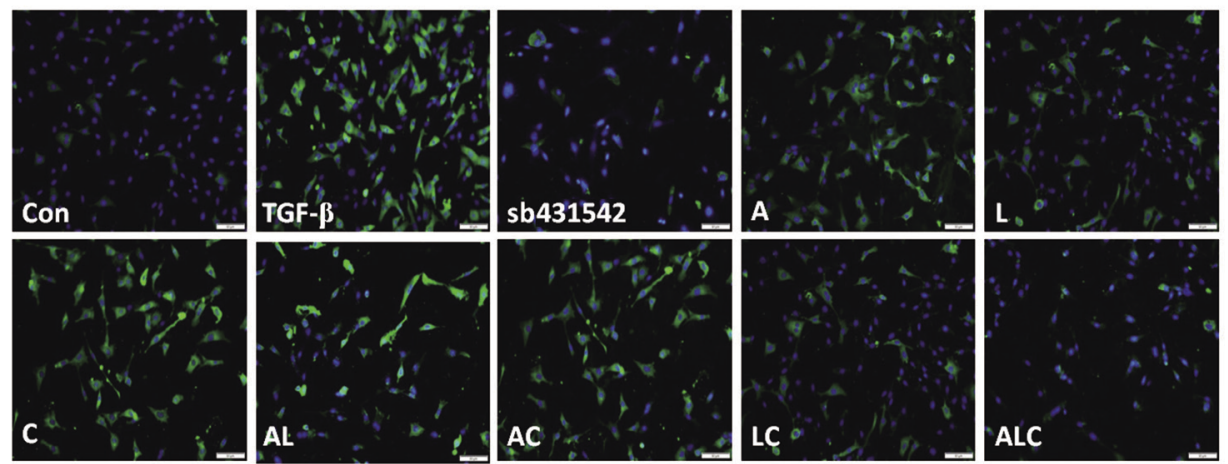

C
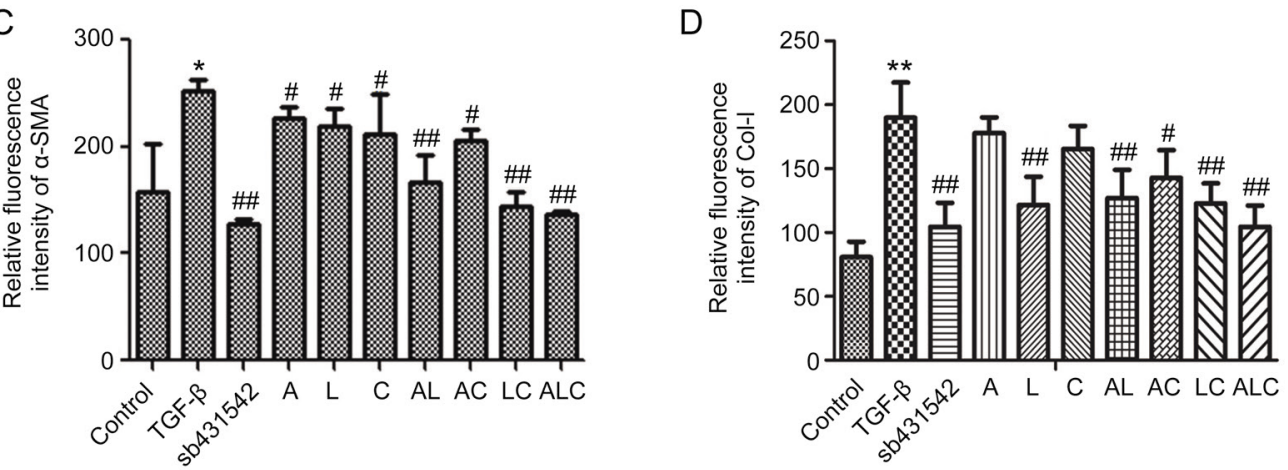

E
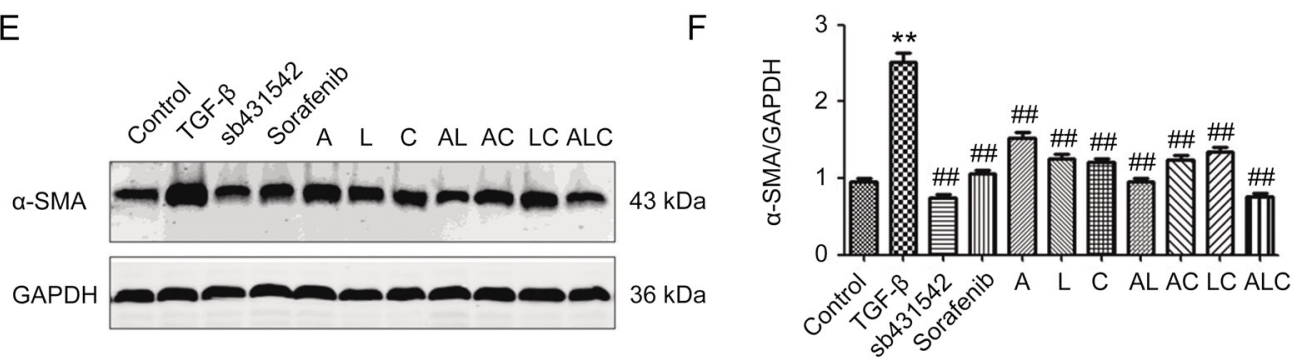

Figure 4. ALC formula decreased the expression of $\alpha-S M A$ and collagen I in LX-2 cells. (A) Fluorescence intensity of $\alpha$-SMA by High Content Screening. (B) Fluorescence intensity of collagen I by High Content Screening. (C) Semi-quantitative analysis of the relative fluorescence intensity of $\alpha$-SMA. (D) Semiquantitative analysis of the relative fluorescence intensity of collagen I. (E) Western blot assay of $\alpha$-SMA in LX-2 cells. (F) Semi-quantitative analysis of $\alpha$-SMA level. ${ }^{*} P<0.05,{ }^{* *} P<0.01$ vs control group. ${ }^{\#} P<0.05,{ }^{\# \#} P<0.01$ vs model group.

The ALC formula decreased expression of $\alpha$-SMA in liver tissues of DMN-treated C57BL/6 mice model

To investigate the mechanism of the ALC formula, a-SMA expression, a marker of hepatic stellate cell (HSC) activation in DMN-treated mice liver tissue, was determined by Western blotting. As shown in Figure 5E and F, DMN group mice showed significantly increased expression of a-SMA compared to the control group. The a-SMA expression level was 
almost 2.5-fold higher in the model group than in the normal group. However, the ALC formula, which functions similarly to sorafenib, resulted in a more marked decrease than in the model group (Figure 5E and F).

\section{Discussion and conclusion}

Liver fibrosis is a chronic liver disease that exhibits excessive accumulation of extracellular matrix proteins, including collagen. The main causes of liver fibrosis include chronic HBV, HCV infection, alcohol abuse, and nonalcoholic steatohepatitis $(\mathrm{NASH})^{[31]}$. Liver fibrosis is an essential pathological process in chronic liver disease and can lead to cirrhosis. Therefore, it is very important to find an approach to reverse or regress liver fibrosis. Liver fibrosis is believed to occur by activation of stellate cells within the liver into an activated myofibroblast phenotype, resulting in cell proliferation, a-smooth muscle actin (a-SMA) expression, and collagen deposition ${ }^{[32]}$. Activation of HSCs leads to retinoid storage, remodeling of the ECM and production of growth factors and cytokines. Suppression of HSC activation has been suggested to be a therapeutic target against hepatic fibrosis ${ }^{[33]}$. Sorafenib, a multiple receptor tyrosine kinase inhibitor targeting the platelet-derived growth factor (PDGF) receptor and the Raf/extracellular-signalregulated kinase (ERK) signaling pathway, has been shown to induce suppression of collagen accumulation and HSCs growth. Sorafenib can be used as a potential therapeutic agent for the treatment of liver fibrosis ${ }^{[34-36]}$.

DBT is a classic formula from Chinese traditional medicine that has been widely used for the treatment of liver, kidney, lung and other organ fibrosis ${ }^{[8-12,37-39]}$. However, DBT is a very complex mixture containing hundreds of components. Numerous studies have shown that DBT consists of volatile compounds, phthalides, organic acids, triterpene saponins and flavonoids ${ }^{[13-21]}$. Traditional Chinese medicines, due to longterm clinical testing and their reliable therapeutic efficacy, are attracting increased global attention as excellent pools of bioactive compounds for the discovery of new drugs. However, the large numbers of other components in traditional Chinese medicines make screening and analysis of bioactive components extremely difficult ${ }^{[40]}$. It is believed that, at least in some formulae, multiple components could hit multiple targets and exert a synergistic therapeutic efficacy. However, the essential compounds have not been identified in most formulae and the precise mechanisms of formulae remain to be addressed using
A

$\mathrm{B}$
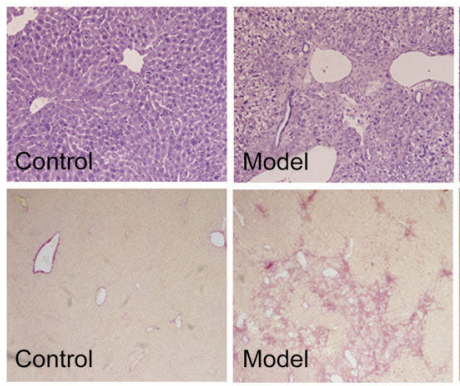

C

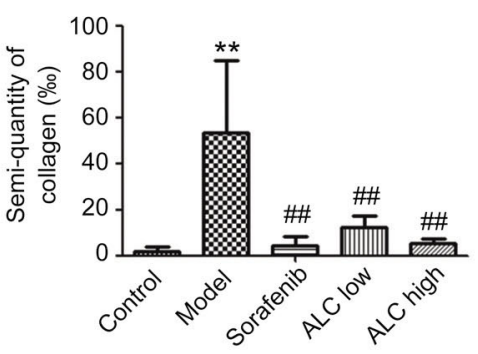

E

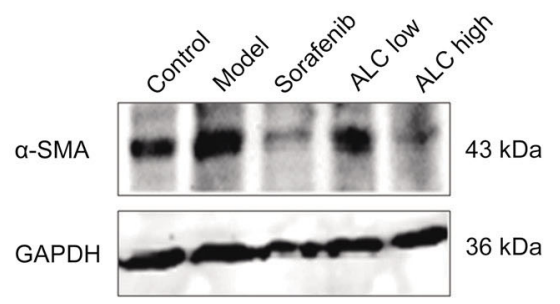

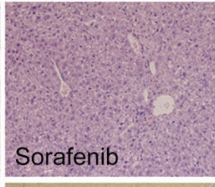
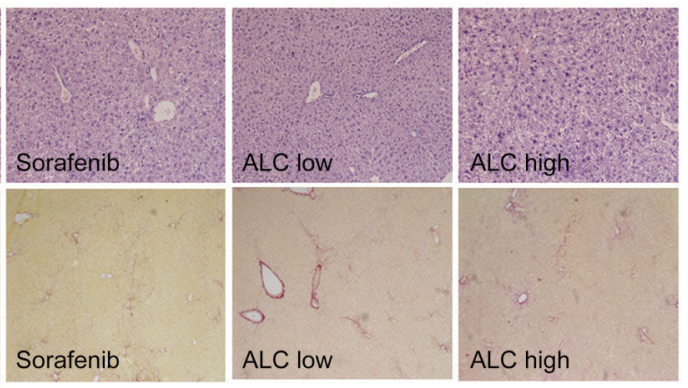

$\mathrm{D}$

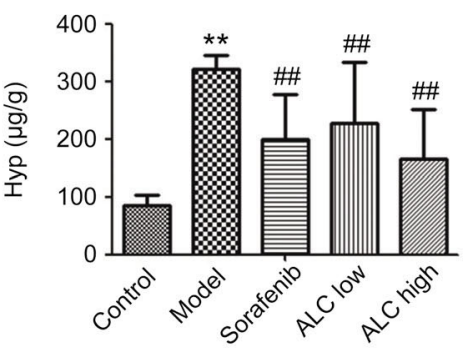

$\mathrm{F}$

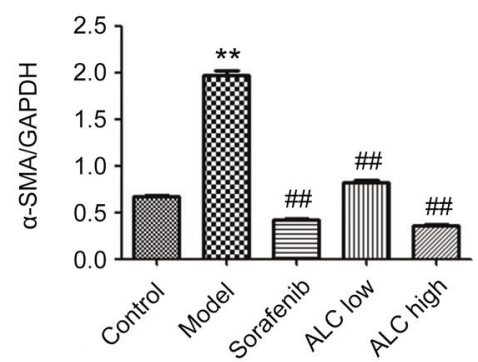

Figure 5. ALC formula reduced collagen deposition and Hyp content in mice liver. (A) H\&E staining, $\times 100$. (B) Hepatic Sirius-Red staining, $\times 100$. (C) Semi-quantitative analysis of hepatic collagen. (D) Assessment of hepatic Hyp. (E) Western blot analysis revealed $\alpha-S M A$ expression in mice liver. (F) Semi-quantitative analysis of $\alpha$-SMA level. ${ }^{* *} P<0.01$ vs control group. ${ }^{\# \#} P<0.01$ vs model group. 
molecular approaches, thus hampering the modernization of $\mathrm{TCM}^{[23]}$. Therefore, the exploration of effective components of DBT is very meaningful and may produce novel drugs.

The present study demonstrated that astragaloside I, levistilide $\mathrm{A}$ and calycosin may be the three main active anti-hepatic fibrosis components from DBT. They can inhibit activation of hepatic stellate cell line LX-2, which can be induced by TGF- $\beta 1$. The Chou-Talalay method was employed to explore their synergistic effect on activated LX-2 cells, and it was shown that the ALC could inhibited proliferation of LX-2 and decreased expression of a-SMA and collagen I in LX-2 cells (Figure 4).

DMN increases the levels of serum biochemical parameters, including ALT and AST, which are parallel to liver damage ${ }^{[41]}$. The increase in AST and ALT reflects hepatocellular injury. In this study, treatment with the ALC formula markedly reduced the increased levels of AST and ALT. The Hyp content assay revealed that compared to normal mice, model mice showed a significant increase in Hyp content. The ALC formula at both a high and a low dose significantly decreased the hepatic levels of Hyp $(P<0.01)$ (Figure 5D). These results revealed that treatment with the ALC formula efficiently reversed liver fibrosis processes in the presence of DMN.

Histological assessment indicated a protective effect of the ALC formula against DMN-induced liver fibrosis. A normal liver has a lobular architecture with a central vein and radiating hepatic cords, but in liver fibrosis, broad infiltration of lymphocytes appears around the central vein together with marked collagen deposition. In the histopathological evaluation, severe structural damage, formation of dense fibrotic septa and proliferation of bile ducts in the presence of inflammatory cells were observed. Treatment with sorafenib and the ALC formula recovered the liver structure from fibrosis (Figure 5).

Activation of HSCs represents the key initial step in the pathogenesis of liver fibrosis. Expression of a-SMA is a representative feature of activated HSCs and is considered to be a hallmark for liver fibrosis ${ }^{[1-3]}$. In this study, we measured the expression of a-SMA by immunofluorescence staining and western blot analysis in LX-2 cells and found that ALC also had a potent synergistic inhibitory effect on the fluorescence intensity of a-SMA in LX-2 cells. Furthermore, an in vivo assay revealed that the ALC formula significantly decreased the a-SMA expression level in liver tissues compared to the DMN group, in accordance with the above results of in vitro assays.

In conclusion, the present study established that astragaloside I, levistilide A and calycosin may be the main three active anti-hepatic fibrosis components from DBT, and this novel combination, the "ALC formula," has potent anti-fibrosis effects in vitro and in vitro. The ALC formula inhibited proliferation in LX-2 and decreased expression of a-SMA and collagen I in LX-2 cells. It significantly reduced collagen deposition, the hydroxyproline content and the a-SMA expression levels in liver tissues compared to the DMN group.

\section{Acknowledgments}

This work was supported by grants from the National Natural
Science Foundation of China (№ 81402515 and 81473404).

We thank Dr Jing LYU for providing the methods and other helps.

\section{Author contribution}

Study concept and design: Cheng-hai LIU; biological study: Tao GUO, Zu-long LIU and Qiang ZHAO; analysis of data: Cheng-hai LIU and Zhi-min ZHAO; preparation of the manuscript: Zu-long LIU and Cheng-hai LIU.

\section{References}

1 Lee YA, Wallace MC, Friedman SL. Pathobiology of liver fibrosis: a translational success story. Gut 2015; 64: 830-41.

2 Wynn TA, Ramalingam TR. Mechanisms of fibrosis: therapeutic translation for fibrotic disease. Nat Med 2012; 18: 1028-40.

3 Mederacke I, Dapito DH, Affò S, Uchinami H, Schwabe RF. High-yield and high-purity isolation of hepatic stellate cells from normal and fibrotic mouse livers. Nat Protocols 2015; 10: 305-15.

4 Lee JH, Jang EJ, Seo HL, Ku SK, Lee JR, Shin SS, et al. Sauchinone attenuates liver fibrosis and hepatic stellate cell activation through TGF-ß/Smad signaling pathway. Chem Biol Interact 2014; 224: 5867.

5 Zhang L, Schuppan D. Traditional Chinese Medicine (TCM) for fibrotic liver disease: hope and hype. J Hepatol 2014; 61: 166-68.

6 Lv J, Zhao Z, Chen Y, Wang Q, Tao Y, Yang L, et al. The chinese herbal decoction danggui buxue tang inhibits angiogenesis in a rat model of liver fibrosis. Evid Based Complement Alternat Med 2012; 224: 58-67.

7 Chen Y, Chen Q, Lu J, Li FH, Tao YY, Liu CH. Effects of Danggui Buxue Decoction on lipid peroxidation and MMP-2/9 activities of fibrotic liver in rats. Chin J Integr Med 2009; 15: 435-41.

8 Wang WL, Sheu SY, Chen YS, Kao ST, Fu YT, Kuo TF, et al. Evaluating the bone tissue regeneration capability of the Chinese herbal decoction Danggui Buxue Tang from a molecular biology perspective. Biomed Res Int 2014; 2014: 853234.

9 Zierau O, Zheng KY, Papke A, Dong TT, Tsim KW, Vollmer G. Functions of Danggui Buxue Tang, a Chinese Herbal Decoction containing Astragali Radix and Angelicae Sinensis Radix, in uterus and liver are both estrogen receptor-dependent and -independent. Evid Based Complement Alternat Med 2014; 2014: 438531.

10 Gong AG, Li N, Lau KM, Lee PS, Yan L, Xu ML, et al. Calycosin orchestrates the functions of Danggui Buxue Tang, a Chinese herbal decoction composing of Astragali Radix and Angelica Sinensis Radix: An evaluation by using calycosin-knock out herbal extract. J Ethnopharmacol 2015; 168: 150-7.

11 Zhao P, Zhou WC, Li DL, Mo XT, Xu L, Li LC, et al. Total glucosides of Danggui Buxue Tang attenuate BLM-induced pulmonary fibrosis via regulating oxidative stress by inhibiting NOX4. Oxid Med Cell Longev 2015; 2015: 645814.

12 Ye TS, Zhang YW, Zhang XM. Protective effects of Danggui Buxue Tang on renal function, renal glomerular mesangium and heparanase expression in rats with streptozotocin-induced diabetes mellitus. Exp Ther Med 2016; 11: 2477-83.

13 Yi L, Qi LW, Li P, Ma YH, Luo YJ, Li HY. Simultaneous determination of bioactive constituents in Danggui Buxue Tang for quality control by HPLC coupled with a diode array detector, an evaporative light scattering detector and mass spectrometry. Anal Bioanal Chem 2007; 389: 571-80.

14 Zhang WL, Zheng KY, Zhu KY, Zhan JY, Bi CW, Chen JP, et al. Chemical and biological assessment of Angelica herbal decoction: comparison of different preparations during historical applications. Phytomedicine 
2012; 19: 1042-8.

15 Lin LZ, He XG, Lian LZ, King W, Elliott J. Liquid chromatographicelectrospray mass spectrometric study of the phthalides of Angelica sinensis and chemical changes of Z-ligustilide. J Chromatogr A 1998; 810: 71-9.

16 Lao S, Li S, Kan KK, Li P, Wan J, Wang Y, et al. Identification and quantification of 13 components in Angelica sinensis (Danggui) by gas chromatography-mass spectrometry coupled with pressurized liquid extraction. Anal Chim Acta 2004; 526: 131-7.

17 Dong TT, Zhao KJ, Gao QT, Ji ZN, Zhu TT, Li J, et al. Chemical and biological assessment of a Chinese herbal decoction containing Radix Astragali and Radix Angelicae Sinensis: determination of drug ratio in having optimized properties. J Agric Food Chem 2006; 54: 2767-74.

18 Wang P, Liang YZ. Chemical composition and inhibitory effect on hepatic fibrosis of Danggui Buxue Decoction. Fitoterapia 2010; 81: 793-8.

19 Chan PH, Zhang WL, Cheung CY, Tsim KW, Lam H. Quality control of Danggui Buxue Tang, a Traditional Chinese Medicine Decoction, by ${ }^{1} \mathrm{H}$-NMR metabolic profiling. Evid Based Complement Alternat Med 2014; 2014: 567893.

20 Shi X, Tang Y, Zhu H, Li W, Li W, Li Z, et al. Pharmacokinetic comparison of seven major bio-active components in normal and blood deficiency rats after oral administration of Danggui Buxue decoction by UPLC-TQ/MS. J Ethnopharmacol 2014; 153: 169-77.

21 Zhang YZ, Xu F, Yi T, Zhang JY, Xu J, Tang YN, et al. Chemical profile analysis and comparison of two versions of the classic TCM formula Danggui Buxue Tang by HPLC-DAD-ESI-IT-TOF-MSn. Molecules 2014; 19: 5650-73.

22 Yuan Y, Zong J, Zhou H, Bian Z-Y, Deng W, Dai J, et al. Puerarin attenuates pressure overload-induced cardiac hypertrophy. J Cardiol 2014; 63: 73-81.

23 Wang L, Zhou GB, Liu P, Song JH, Liang Y, Yan XJ, et al. Dissection of mechanisms of Chinese medicinal formula Realgar-Indigo naturalis as an effective treatment for promyelocytic leukemia. Proc Natl Acad Sci U S A 2008; 105: 4826-31.

24 Chou TC. Drug combination studies and their synergy quantification using the Chou-Talalay method. Cancer Res 2010; 70: 440-6.

25 Chou TC. Combined treatment of pancreatic cancer with mithramycin A and tolfenamic acid promotes Sp1 degradation and synergistic antitumor activity-Letter. Cancer Res 2011; 71: 2793-93.

26 Eid SY, El-Readi MZ, Wink M. Synergism of three-drug combinations of sanguinarine and other plant secondary metabolites with digitonin and doxorubicin in multi-drug resistant cancer cells. Phytomedicine 2012; 19: 1288-97.

27 Jamall IS, Finelli V, Hee SQ. A simple method to determine nanogram levels of 4-hydroxyproline in biological tissues. Anal Biochem 1981; 112: $70-5$.

28 Gressner A, Weiskirchen R. Modern pathogenetic concepts of liver fibrosis suggest stellate cells and TGF- $\beta$ as major players and therapeutic targets. J Cell Mol Med 2006; 10: 76-99.

29 Kisseleva T, Brenner DA. Hepatic stellate cells and the reversal of fibrosis. J Gastroenterol Hepatol 2006; 21 (s3).

30 Tsukada S, Parsons CJ, Rippe RA. Mechanism of liver fibrosis. Clin Chim Acta 2006; 364: 33-60.

31 Elpek GÖ. Cellular and molecular mechanisms in the pathogenesis of liver fibrosis: an update. World J Gastroenterol 2014; 20: 7260.

32 Seki E, Brenner DA. Recent advancement of molecular mechanisms of liver fibrosis. J Hepatobiliary Pancreat Sci 2015; 22: 512-8.

33 Zhou DJ, Mu D, Jiang MD, Zheng SM, Zhang Y, He S, et al. Hepatoprotective effect of juglone on dimethylnitrosamine-induced liver fibrosis and its effect on hepatic antioxidant defence and the expression levels of $\alpha$-SMA and collagen III. Mol Med Rep 2015; 12 : 4095-102.

34 Wang Y, Gao J, Zhang D, Zhang J, Ma J, Jiang $\mathrm{H}$. New insights into the antifibrotic effects of sorafenib on hepatic stellate cells and liver fibrosis. J Hepatol 2010; 53: 132-44.

35 Thabut D, Routray C, Lomberk G, Shergill U, Glaser K, Huebert R, et al. Complementary vascular and matrix regulatory pathways underlie the beneficial mechanism of action of sorafenib in liver fibrosis. Hepatology 2011; 54: 573-85.

36 Deng YR, Ma HD, Tsuneyama K, Yang W, Wang YH, Lu FT, et al. STAT3mediated attenuation of $\mathrm{CCl}_{4}$-induced mouse liver fibrosis by the protein kinase inhibitor sorafenib. J Autoimm 2013; 46: 25-34.

37 Gao J, Huang Y, Li P, Xu D, Li J, Liu Y, et al. Antifibrosis effects of total glucosides of Danggui-Buxue-Tang in a rat model of bleomycininduced pulmonary fibrosis. J Ethnopharmacol 2011; 136: 21-6.

38 Gao J, Feng Lj, Huang Y, Li P, Xu Dj, Li J, et al. Total glucosides of Danggui Buxue Tang attenuates bleomycin-induced pulmonary fibrosis via inhibition of extracellular matrix remodelling. J Pharm Pharmacol 2012; 64: 811-20.

39 Wei MG, Sun W, Xiong PH, Shao JD. Antifibrotic effect of the Chinese herbs Modified Danggui Buxue Decoction on adriamycin-induced nephropathy in rats. Chin J Integr Med 2012; 18: 591-8.

40 Huang X, Kong L, Li X, Chen X, Guo M, Zou H. Strategy for analysis and screening of bioactive compounds in traditional Chinese medicines. J Chromatogr B 2004; 812: 71-84.

41 Islam S, Antonsson L, Westin J, Lagging M. Cirrhosis in hepatitis C virus-infected patients can be excluded using an index of standard biochemical serum markers. Scandinavian J Gastroenterol 2005; 40: 867-72. 